\title{
Establishing an osteoimmunomodulatory coating loaded with aspirin on the surface of titanium primed with phase-transited lysozyme
}

This article was published in the following Dove Medical Press journal: International Journal of Nanomedicine

\author{
Wenxin Zhang' \\ $X$ in $\mathrm{Lu}^{\prime}$ \\ Zuoying Yuan ${ }^{2}$ \\ Minjuan Shen' \\ Yunjia Song' \\ Huanhuan Liu' \\ Jingjing Deng' \\ Xue Zhong' \\ Xu Zhang'
}

'School of Dentistry, Stomatological Hospital, Tianjin Medical University, Tianjin, People's Republic of China; ${ }^{2}$ State Key Laboratory of OrganicInorganic Composites, Beijing Laboratory of Biomedical Materials, Beijing University of Chemical Technology, Beijing, People's Republic of China
Correspondence: Xu Zhang School of Dentistry, Hospital of Stomatology, Tianjin Medical University, 12 Observatory Road, Tianjin 300070, People's Republic of China

Tel +86 I39 20376897

Fax +862223332016

Email zhangxu@tmu.edu.cn
Background: To improve osseointegration and enhance the success rate of implanted biomaterials, the surface modification technology of bone implants has developed rapidly. Intensive research on osteoimmunomodulation has shown that the surfaces of implants should possess favorable osteoimmunomodulation to facilitate osteogenesis.

Methods: A novel, green and efficient phase-transited lysozyme (PTL) technique was used to prime titanium discs with a positive charge. In addition, sodium hyaluronate (HA) and selfassembled type I collagen containing aspirin (ASA) nanoparticles were decorated on PTL-primed Ti discs via electrostatic interaction.

Results: The behaviors of bone marrow stromal cells (BMSCs) on the Ti disc surfaces containing ASA were analyzed in different conditioned media (CM) generated by macrophages. Additionally, the secretion of inflammation-related cytokines of macrophages on the surfaces of different Ti discs was investigated in in vitro experiments, which showed that the Ti surface containing ASA not only supported the migration, proliferation and differentiation of BMSCs but also reduced the inflammatory response of macrophages compared with Ti discs without surface modification. After implantation in vivo, the ASA-modified implant can significantly contribute to bone formation around the implant, which mirrors the evaluation in vitro.

Conclusion: This study highlights the significant effects of appropriate surface characteristics on the regulation of osteogenesis and osteoimmunomodulation around an implant. Implant modification with ASA potentially provides superior strategies for the surface modification of biomaterials.

Keywords: titanium surface modification, nanoparticle, osteoimmunomodulation, osseointegration

\section{Introduction}

Implantable titanium (Ti) medical devices, such as knee, hip, and dental implants, have been well developed and widely adopted to replace damaged joint tissues and missing teeth and to restore their functions. Although Ti and its alloys exhibit superior biocompatibility, surface modifications are required to improve osseointegration and to enhance the success rate of implants. ${ }^{1}$ So far, most studies have focused on the establishment of coatings with favorable osteogenesis, angiogenesis, and antibacterial abilities. $^{2-4}$ However, inconsistent results between in vitro and in vivo studies have suggested that the capacities of the material mentioned above are insufficient for the mediation of osteogenesis. ${ }^{5,6}$ This can be attributed to the neglect of an initial inflammatory response to the implanted foreign body and osseointegration, which starts from an inflammation-driven process between the extraneous implants and the bone tissue. ${ }^{7}$ 
The immune and skeletal systems are closely related and share numerous cytokines, receptors, signaling molecules, and transcription factors. ${ }^{8,9}$ In innate immunity, macrophages are the center of the metabolism microenvironment, which includes bone defects and exogenous biological materials. ${ }^{10-12}$ Therefore, a new generation of bone implant materials should include multifunctional implants with surfaces that not only are functionalized with osteogenesis and antibacterial properties but also coordinate immunomodulation. With an increased attention on the concept of osteoimmunomodulation, the interaction between implants and host immunity has been studied more thoroughly. ${ }^{13}$

Recent studies have shown that drugs such as nonsteroidal anti-inflammatory drugs (NSAIDs), low-dose doxycycline, bisphosphonates (BPs), and $\omega-3$ fatty acids (anti-inflammatory lipids) are effective in modulating host immune response. ${ }^{14-17}$ However, some scholars have suggested that the long-term use of certain drugs, eg, BPs, has risks; ${ }^{18}$ therefore, the safe and effective use of drugs for immune regulation is also the focus of the current research. Aspirin (ASA), a NSAID, has been widely used for a hundred years to relieve from fever, pain, and inflammation with very low toxic side effects. ${ }^{19,20}$ In recent years, increasingly more studies have found that ASA may affect the balance of bone metabolism and exhibit dose dependence. ${ }^{21-24}$ In addition, ASA has been proved to enhance osteogenic differentiation and to exert an anti-inflammatory effect through certain biological pathways. ${ }^{5,14,25,26}$ Thus, ASA loading onto the surface of implants can endow the material surface with immunomodulatory properties. However, investigations into both the effective loading of ASA onto the surface of implants and the control of its release are lacking.

Until now, microspheres and layer-by-layer self-assembly techniques have been used to modify the surfaces of implants to control the burst release of target drugs to some extent. ${ }^{27}$ At present, ASA-loaded chitosan nanoparticles (ACS) have been prepared and have proved to have a good sustained release effect; ${ }^{28}$ however, few studies have reported the immobilization of these nanoparticles on the surfaces of $\mathrm{Ti}$ implants to regulate immunomodulation.

A novel phase-transited lysozyme (PTL) technique was used to form a proteinaceous coating on the surfaces of implants. ${ }^{29}$ Under physiological conditions, lysozyme can form nanostructured amyloid fibers with a similar cross- $\beta$ sheet internal structure under the action of a reducing agent. ${ }^{30}$ Those fibers can firmly and quickly attach onto various substrate surfaces regardless of the substrate type. ${ }^{31}$ As this coating can be prepared efficiently with a controllable thickness, the PTL coating is a desirable surface priming method for advanced materials. The resulting PTL coating confers surfaces with positively charged groups, providing an active interface for further functionalization. ${ }^{32}$ The PTL coating can directly bind $\mathrm{Ca}^{2+}$ ions via abundant carboxyl groups that nucleate and induce the formation of a hydroxyapatite layer on the implant surfaces, thus improving the osteoconductivity and osteoinductivity of implants. ${ }^{33}$ Furthermore, the initial layer of PTL is able to connect polyelectrolyte multilayers (PEMs) loaded with antibacterial agents, osteogenic growth factors, cytokines, and/or other functional components to Ti surfaces, thereby preventing implant-associated infection and facilitating osseointegration in the early stage of implantation. ${ }^{34,35}$

Accordingly, it was hypothesized that ASA-loaded multilayers could be fabricated on the surfaces of implants primed with PTL coating, which would confer relatively antiinflammatory efficacy and attenuate host immune response on implants, ultimately promoting osteogenic property of the $\mathrm{Ti}$ surface. In this study, a PTL nanofilm was primed on the surface of pure Ti discs, providing a positively charged surface that lays the foundation for subsequent experiments. Then, PEMs, sodium hyaluronate (HA, negatively charged), and self-assembled type I collagen (COL I, positively charged) were successively coated on the PTL layer via electrostatic attractions. The self-assembled COL I not only acts as a dispersing agent for nanoparticles but also simulates the extracellular matrix of attached bone cells and promotes cell growth. ${ }^{36-38}$ The test was performed to confirm the impact of our multifaceted coating on osteogenesis, followed by the evaluation of osteoimmunomodulatory properties.

\section{Materials and methods Specimen preparation}

The pure Ti discs (99.6\% purity; Leiden Biomaterials Co., Shanghai, People's Republic of China) with a dimension of $14.75 \times 2.0 \mathrm{~mm}$ (diameter $\times$ thickness) for biological experiments and pure Ti rods (99.6\% purity) with a dimension of $2.0 \times 2.0 \mathrm{~mm}$ were polished by $\mathrm{SiC}$ sandpaper to 8,000 grit; sequentially cleaned in acetone, ethanol, and deionized water; dried; and then sterilized in an autoclave at $120^{\circ} \mathrm{C}$ for 1 hour.

\section{Fabrication of PTL-primed Ti substrates}

To functionalize the sample surfaces with a positive charge, the lysozyme phase transition solution prepared by a simple mixing of an equivolume solution of lysozyme $(2 \mathrm{mg} / \mathrm{mL}$, in $10 \mathrm{mM}$ of 4-(2-hydroxyethyl)-1-piperazineethanesulfonic acid [HEPES] buffer, $\mathrm{pH}=7.4$ ) and tris(2-carboxyethyl) phosphine (TCEP; $50 \mathrm{mmol}$, in $10 \mathrm{mM}$ of HEPES buffer, $\mathrm{pH}=6.0$, adjusted the $\mathrm{pH}$ by $5 \mathrm{M} \mathrm{NaOH}$ ) was placed 
dropwise on the polished $\mathrm{Ti}$ discs/rods mentioned above. After incubation for 50 minutes at room temperature, these Ti discs/rods were washed several times with ultrapure water to remove residual impurities and dried with nitrogen gas.

\section{Preparation of ACS and self-assembled collagen}

In the first stage, chitosan was dissolved in $1 \%(\mathrm{v} / \mathrm{v})$ aqueous acetic acid to obtain a $1 \mathrm{mg} / \mathrm{mL}$ chitosan solution. Various amounts of anhydrous ethanol-saturated solutions of ASA were individually added to the $25 \mathrm{~mL}$ acidic chitosan solution. Subsequently, $0.75 \mathrm{mg} / \mathrm{mL}$ aqueous tripolyphosphate (TPP) solution was added dropwise to the chitosan-ASA solution under vigorous magnetic stirring. The addition of TPP was stopped when the solution became pale blue in color; stirring was continued, and the reaction was maintained for 30 minutes. The final mixture was centrifuged at 1,500 r/min for 30 minutes. Finally, the ACS were washed repeatedly with distilled water and then placed in a refrigerator at $4^{\circ} \mathrm{C}$ for future use.

The method for extracting rat tail for this experiment was derived from a previous study. ${ }^{39}$ The extracted collagen solution was centrifuged at $4^{\circ} \mathrm{C}$ and $3,000 \mathrm{r} / \mathrm{min}$ for 30 minutes, and the supernatant solution after centrifugation was transferred into a dialysis bag, which was then dialyzed in $0.02 \mathrm{M}$ PBS for 5 days to complete the collagen self-assembly process. In addition, the prepared ACS were then added to the collagen solution to form a dispersion solution.

\section{Fabrication of multilayer coatings on the surfaces of PTL-primed Ti substrates}

To uniformly deposit multilayer coatings on the surfaces of the PTL-activated Ti discs, the discs were dipped in each polyelectrolyte solution for 15 minutes. The substrate was sequentially treated with anionic HA solution $(1 \mathrm{mg} / \mathrm{mL}$ in $0.14 \mathrm{M}$ aqueous $\mathrm{NaCl}, \mathrm{pH}=6.0$ ) and then covered by the selfassembled collagen/ACS mixture. Each dip was followed by washing with $0.14 \mathrm{M} \mathrm{NaCl}$ for 5 minutes. Then, the samples were rinsed twice for 30 minutes in ultrapure water, air-dried, and sterilized using a $25 \mathrm{kGy}$ dose of $\gamma$ radiation (cobalt-60; Huanming Gaoke Fuzhao Co., Tianjin, People's Republic of China). Finally, these functionalized $\mathrm{Ti}$ discs were stored at $50 \% \pm 5 \%$ relative humidity before follow-up experiments.

\section{Characterization}

The nanoparticles were uniformly immersed in collagen solution and placed dropwise on copper grids (400 mesh size), dried at room temperature, and then examined using transmission electron microscopy (TEM; JEM-2100F; JEOL, Tokyo, Japan). The surface microstructure morphology of the pristine and decorated $\mathrm{Ti}$ discs was evaluated by fieldemission scanning electron microscopy (SEM; Nova Nano SEM 430; FEI, Hillsboro, OR, USA) with a beam voltage of $15 \mathrm{kV}$. All the samples were sputter-coated with gold before the SEM observation. X-ray photoelectron spectroscopy (XPS; AXIS His; Kratos Analytical Ltd., Manchester, UK) was used to identify the chemical constituents of pristine and the modified Ti disc surfaces. The contact angle of each layer with simulated body fluid (SBF) was measured using a drop-shape analysis system (JC2000D1; Micaren, Fujian, People's Republic of China) at room temperature. The mean static contact angle of each Ti surface was determined from measurements taken in triplicate. Each Ti disc was measured in triplicate to calculate the mean values.

\section{In vitro release of ASA from the prepared samples}

To analyze the release profile of ASA, Ti discs $(n=3)$ containing different concentrations of $\operatorname{ACS}(0.5,1.0,1.5,3.0$, and $5.0 \mathrm{~mL}$ ) were immersed in deionized water in 24-well plates and incubated at $37^{\circ} \mathrm{C}$ and $5 \% \mathrm{CO}_{2}$ under $100 \%$ humidity. At various time points $(1,2,3,4,5,6$, and 7 days), the supernatant was collected, and $1 \mathrm{~mL}$ samples were removed and replaced with the same volume of fresh medium. The amount of ASA released into the supernatant was quantified using ultraviolet spectrophotometry (BioSpectrometer ${ }^{\mathbb{R}}$; Eppendorf, Hamburg, Germany) at $280 \mathrm{~nm}(\mathrm{n}=3)$. A standard curve was used to determine the release of ASA at different concentrations.

\section{Proliferation and differentiation of BMSCs on different samples}

The Ti samples $(n=3)$ containing different concentrations of ACS $(0,1.0,3.0$, and $5.0 \mathrm{~mL})$, pure Ti samples, and TiPTL-HA-COL samples were preplaced in a 24-well plate. Then, $8 \times 10^{3}$ BMSCs, which were purchased from Cyagen Biosciences (Guangzhou, People's Republic of China), in $1,000 \mu \mathrm{L}$ complete growth medium (DMEM with $10 \%$ fetal bovine serum [FBS], $100 \mathrm{mg} / \mathrm{mL}$ streptomycin, and $100 \mathrm{U} / \mathrm{mL}$ penicillin; Invitrogen, Carlsbad, CA, USA) were seeded onto the 24-well plate, which was maintained in a $5 \% \mathrm{CO}_{2}$ incubator at $37^{\circ} \mathrm{C}$.

Cell proliferation of each sample was measured using a Cell Counting Kit-8 (CCK-8) assay (Solarbio, Beijing, People's Republic of China). To determine the cell proliferation of different concentrations of ACS on Ti samples, $900 \mu \mathrm{L}$ of fresh medium was added to each well to replace the original culture medium that was washed with PBS; $90 \mu \mathrm{L}$ of the CCK-8 solution was added to each well at various culturing 
intervals ( 1,3 , and 5 days). The plates were then incubated in the $5 \% \mathrm{CO}_{2}$ incubator at $37^{\circ} \mathrm{C}$ for 2 hours. Subsequently, the samples of the supernatant solutions were transferred into a 96-well plate, and the absorbance of each sample was measured at $450 \mathrm{~nm}$ using a microplate reader (RT-6000; Rayto, Shenzhen, People's Republic of China).

To study the differentiation of the BMSCs incubated with different samples, ALP experiments were performed at various time points using ALP assay kits (NJJ CBIO, Nanjing, People's Republic of China). BMSCs seeded onto different samples were cultured in a fresh medium. After chemical cracking and dilution, aliquots of cell lysates were collected to assess the ALP activity after 7 and 14 days of culture. For normalization, the total protein concentration was measured using a bicinchoninic acid protein assay kit (Solarbio).

\section{Anti-inflammatory activity of RAW264.7 cells on different surfaces}

RAW264.7 cells purchased from Cyagen Biosciences were cultured in DMEM (Invitrogen) with 10\% FBS (EquitechBio, Kerrville, TX, USA). The cells were seeded onto 24-well plates at $1 \times 10^{4}$ cells/well. When the cells were $70 \%-80 \%$ confluent, they were replaced with a medium containing $100 \mathrm{ng} / \mathrm{mL}$ lipopolysaccharides (LPS) for 6 hours. In addition, the induced macrophages were then digested and routinely cultured in a fresh complete growth medium without LPS. Following incubation for 24 hours, the concentrations of inflammatory protein markers, tumor necrosis factor- $\alpha$ (TNF- $\alpha$ ) and nitrite $\left(\mathrm{NaNO}_{2}\right)$, were determined using ELISA kits (R\&D Systems, MN, US), according to the manufacturer's instructions to verify the success of induction.

Then, the cells were seeded onto the prepared Ti discs (blank Ti discs as the blank group and discs containing ASA-loaded nanoparticles [3.0 mL ASA was added in the preparation of ACS] as the ASA group) in 24-well plates at $1 \times 10^{4}$ cells/well. The concentrations of inflammatory protein marker $\left(\mathrm{NaNO}_{2}\right)$ were determined using ELISA kits, and the quantitative PCR (qPCR) analysis was used to detect the mRNA expression of inflammation-related genes (TNF- $\alpha$ and interleukin [IL]-6). Total RNA of the RAW264.7 cells was isolated for 12 hours using the RNAiso Plus system (TaKaRa, Dalian, Japan), and total mRNA was isolated using the TRIzol reagent (Invitrogen) and converted into cDNA using a Revert Aid First-Strand cDNA Synthesis Kit (Thermo Fisher Scientific, Waltham, MA, USA). qPCR was carried out using SYBR Green (Roche, Basel, Switzerland) on an ABI 7500 RT-PCR instrument (Applied Biosystems; Thermo Fisher Scientific); Table 1 lists the primers used.

\section{Behavior of BMSCs on different surfaces in conditioned medium (CM) \\ Collection and preparation of CM}

To prepare the CM for RAW264.7 cells, they were seeded onto the prepared different Ti samples (blank Ti discs as the blank group and discs containing ASA-loaded nanoparticles [3.0 mL ASA was added in the preparation of ACS] as the ASA group) in 24 -well plates at $1 \times 10^{4}$ cells/well and cultured for 1-14 days. The culture supernatant was collected and stored at $4^{\circ} \mathrm{C}$, and it was diluted 1:1 in DMEM containing $5 \%$ FBS before use and denoted as either the blank group or the ASA group. For CM2, the RAW264.7 cells were cultured in a conventional medium, and the culture supernatant was collected as mentioned above.

\section{Adhesion and migration of BMSCs mediated} by different CM in Transwell ${ }^{\circledR}$ systems

To observe the morphology of BMSCs, after 12 and 48 hours of culture in CM, the samples were washed with PBS and fixed in 4\% paraformaldehyde for 30 minutes at room temperature, and then, they were washed five times with PBS. After being blocked with a bovine serum albumin for 1 hour, the samples were washed three more times with PBS and stained with fluorescein isothiocyanate (FITC)-phalloidin. The F-actin and cell nuclei were viewed by confocal laser

Table I Primers used for real-time PCR

\begin{tabular}{|l|l|l|}
\hline & Forward primer sequence $\left(\mathbf{5}^{\prime} \mathbf{-} \mathbf{3}^{\prime} \mathbf{)}\right.$ & Reverse primer sequence $\left(\mathbf{5}^{\prime} \mathbf{- \mathbf { 3 } ^ { \prime } \mathbf { ) }}\right.$ \\
\hline IL-6 & CATGTTCTCTGGGAATCGTGG & TCCAGGTAGCTATGGTACTCC \\
\hline TNF- $\alpha$ & ACTGAACTTCGGGGTGATCG & TGTCTTTGAGATCCATGCCGT \\
\hline COL-I & GCAGACTGGCAACCTCAAGA & CAGGGCCAATGTCTAGTCCG \\
\hline OCN & TAGTGAACAGACTCCGGCGCTA & TGTAGGCGGTCTTCAAGCCAT \\
\hline ALP & TGGACGGTGAACGGGAAAAT & GGAGGCATACGCCATCACAT \\
\hline RUNX2 & CGGACGAGGCAAGAGTTTCA & GGATGAGGAATGCGCCCTAA \\
\hline
\end{tabular}

Abbreviations: COL I, type I collagen; IL-6, interleukin-6; OCN, osteocalcin; RUNX2, runt-related transcription factor 2; TNF- $\alpha$, tumor necrosis factor- $\alpha$. 
scanning microscopy (Leica SP8; Leica Microsystems, Wetzlar, Germany).

BMSCs at P4 were seeded in the upper chamber of 24-well Transwell ${ }^{\circledR}$ plates $\left(1 \times 10^{4}\right.$ cells/chamber, $8.0 \mu \mathrm{m}$ pore size) and cultured in a standard medium. Then, $0.8 \mathrm{~mL}$ aliquots of the different CM of the blank group and those of the ASA group were added in the lower chamber of the 24-well plates and cultured for 12 or 24 hours to facilitate cell migration through the Transwell ${ }^{\circledR}$ membrane. A sterile swab was applied to gently remove the residual BMSCs in the upper chamber, and the cell number of each sample was measured using the CCK-8 kit.

\section{Osteogenic differentiation of BMSCs on different $\mathrm{Ti}$ surfaces in CM2}

BMSCs were seeded on different Ti samples (blank Ti discs as the blank group and discs containing ASA-loaded nanoparticles [3.0 mL ASA was added in the preparation of ACS] as the ASA group) in CM2 before they were collected for follow-up experiments. qPCR analysis was used to detect the mRNA expression of osteogenic genes such as COL I, osteocalcin (OCN), ALP, and runt-related transcription factor 2 (RUNX2). Total RNA of the BMSCs was isolated after 7 or 14 days as mentioned above, and Table 1 lists the primers used.

\section{In vivo implantation and histological analysis}

A total of 24 male Sprague Dawley rats (8-week-old; specific pathogen-free) were obtained from the Lab Animal Center of the Tianjin Medical University. The investigation conformed to the regulations of the experimental animal administration issued by the Ministry of Science and Technology of China (http://www.most.gov.cn), and the protocol was evaluated and accepted by the Institutional Ethics Committee of the Tianjin Medical University before the animal experiments began.

A femoral defect model (2.0 $\mathrm{mm}$ in diameter) was established, and all 24 rats were randomly divided into two groups: 1) the blank group (pure Ti rod) and 2) the ASA group (Ti rod loaded with $3.0 \mathrm{~mL}$ ASA in the preparation of ACS). All the rats were given pentobarbital sodium $(10 \mathrm{mg} / 100 \mathrm{~g}$ body weight; Sigma Aldrich, St Louis, MO, USA) via intraperitoneal injection as general anesthesia and lidocaine for local anesthesia. The right hind limbs of the rats were shaved, and the skin and muscle tissues were then incised. The midshafts of the femora were exposed after a $1.5-\mathrm{cm}$ incision was made through the skin and muscles from the inner side of the distal femur while avoiding any entrance of the articular cavity of the knee. After the exposure of the femur, a 2-mm drill driven by a motorized dental drill $(\Phi=2 \mathrm{~mm})$, which was cooled by the serial addition of normal saline, and the prepared Ti rods with a dimension of $2.0 \times 2.0 \mathrm{~mm}$ (the blank group and the ASA group) were gently screwed in. A 5-0 suture line was used to suture the muscles and skin.

\section{Morphological observation and analysis of bone healing around the implants}

At 3, 7, and 28 days after implantation, the rats were sacrificed, and the femurs with implants were obtained. After fixation in 4\% paraformaldehyde, the samples were fractionally dehydrated in ethanol and embedded in methyl methacrylate resin (Technovit 7200 VLC; Exakt Apparatebau, Norderstedt, Germany). Starting from the major axis, blocks were cut and ground to a final thickness of $\sim 50 \mu \mathrm{m}$ using a cutting-grinding unit (Exakt Apparatebau). The sections were subjected to Van Gieson (VG) staining, and histological images were captured on a Zeiss Microscope (Zeiss Axio Imager M2; Carl Zeiss, Oberkochen, Germany). In each section, the mean value of new bone volume fraction ([bone area/pore area] $\times 100 \%$ ) was calculated for each sample and statistically compared between the groups. Image-Pro Plus software (Media Cybernetics, Rockville, MD, USA) was used for image analysis.

\section{Observation and analysis of inflammatory cell infiltration around the implants}

At 3 and 7 days after implantation, the rats were sacrificed, and the femurs with implants were obtained. After fixation in $4 \%$ paraformaldehyde, the femurs were decalcified in $10 \%$ EDTA solution (Sigma Aldrich) for 45 days, during which the decalcification solution was replaced every 2 days. Then, the samples were dehydrated in ethanol and embedded in paraffin. A cutting unit (Leica Microsystems) was used to obtain $10-\mu \mathrm{m}$ sections. Some of the sections were stained with HE, and others were processed for mouse anti-rat $\mathrm{CD} 68^{+}$primary antibody. The extent of inflammatory cell infiltration and the proportion of $\mathrm{CD}^{2} 8^{+}$cells were analyzed using Image-Pro Plus software.

\section{Statistical analysis}

Experiments were carried out in triplicate. All data are expressed as the mean \pm SD for continuous variables. Significant differences were identified using the ANOVA followed by the Student-Newman-Keuls post hoc test for parametric data and the Kruskal-Wallis test followed by Dunn's multiple 
comparison test for nonparametric data. Differences were considered statistically significant when $P \leq 0.01$.

\section{Results and discussion Establishing ASA-loaded multilayers on Ti discs}

PEMs containing ACS were fabricated on PTL-primed Ti discs using an electrostatic interactions technology, and the self-assembled COL I and HA were modified first (Figure 1).

The SEM images shown in Figure 2B illustrate the morphology of a pure Ti disc, a Ti-PTL disc, a Ti-PTL-HA disc, and a Ti-PTL-HA-(COL-ACS) disc. Under physiological conditions, lysozyme can form nanostructured amyloid fibers with a similar cross- $\beta$-sheet internal structure under the action of a reducing agent depending on solution $\mathrm{pH}$ and lysozyme and TCEP concentrations. ${ }^{40}$ The thickness of the formed amyloid-like nanofilm can precisely be regulated and can stably be immobilized on various substrates regardless of the material type and morphology. ${ }^{29}$ The resulting PTL nanofilm not only confers positive charges but also moderates hydrophilicity to the material substrate; it also enhances the corrosion resistance of the material surface and contains polar and nonpolar group, and it is thus expected to provide an abundance of active sites for further chemical modification. ${ }^{29,32}$ In fact, the PTL self-assembles into an amyloid-like product, which exists in two forms in the system: a micro-nano-necklace-like network structure and a nanofilm in both gas-liquid interfaces and solid-liquid interfaces. ${ }^{41}$ One of the protein-assembled oligomers had a diameter of $0.5-1 \mu \mathrm{m}$ and formed a necklace-like microfiber network attached to the surface; another form of PTL is a macroscopic two-dimensional nanofilm stably fixed to the sample. Therefore, under the combination of hydrogen bonding and electrostatic interaction, PTL nanofilm can stably adhere to the Ti surface. In this study, an in situ growth method was used to prepare the PTL nanofilms; that is, the surface modification was carried out to lay the foundation for subsequent reactions.

XPS was used to determine the chemical compositions of the different surfaces of the samples, as shown in Figure 2D. All binding energies were referenced to the $\mathrm{C}$ 1s spectrum peak $(284.8 \mathrm{eV})$ as an internal reference after calibrating peak positions. The spectrum of pure Ti showed that the main components included C 1s, Ti 2p3 (458.64 eV), O 1s (530 eV), and $\mathrm{N} 1 \mathrm{~s}(402 \mathrm{eV})$. The rise of $\mathrm{S} 2 \mathrm{p}(165 \mathrm{eV})$ and $\mathrm{P} 2 \mathrm{p}$ $(133 \mathrm{eV})$ peaks, derived from (TCEP), and the significantly lower peak of Ti 2p3 indicates successful immobilization

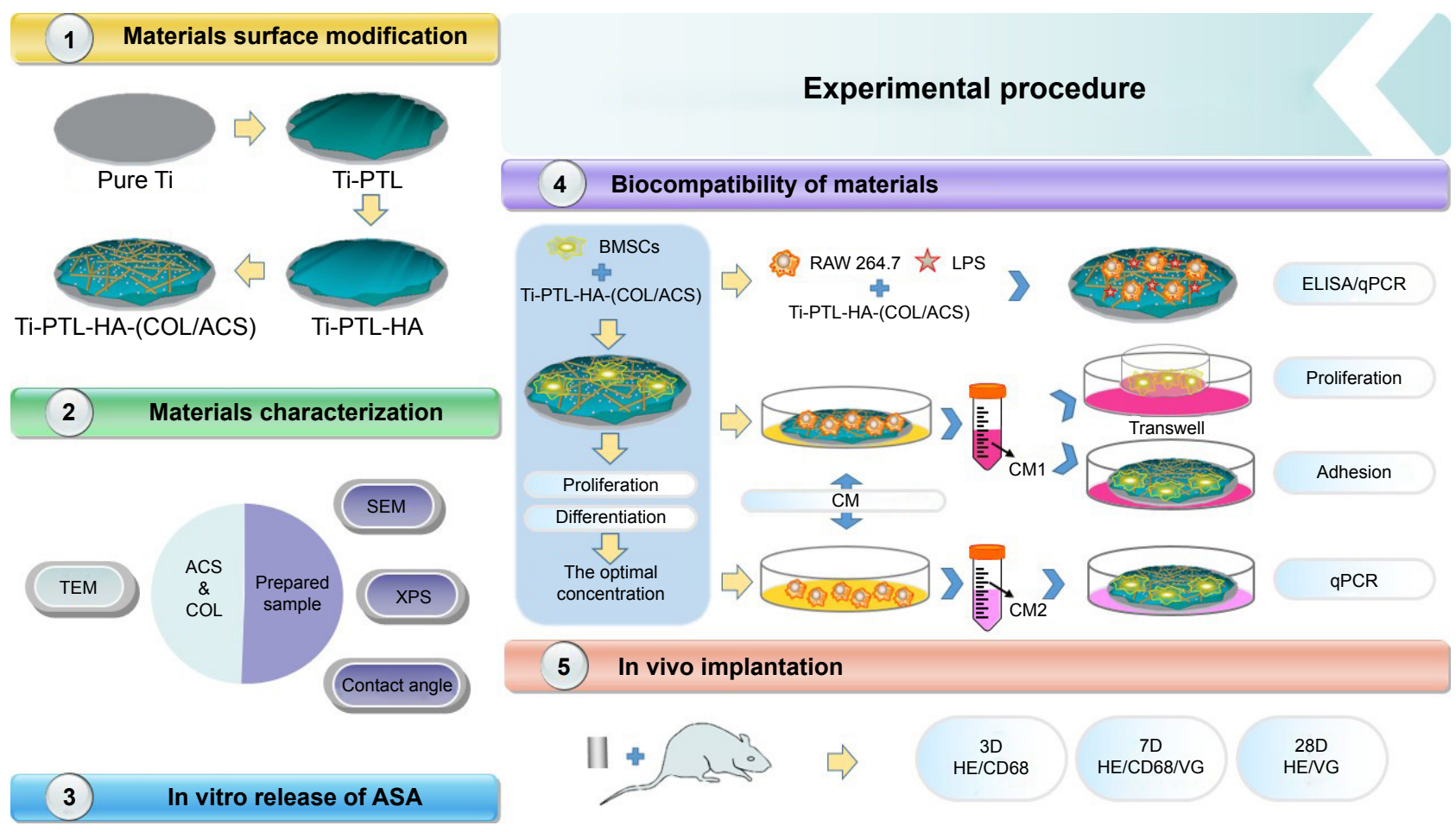

Figure I Flow diagram of the experimental procedure.

Abbreviations: ACS, ASA-loaded chitosan nanoparticles; ASA, aspirin; BMSCs, bone marrow stromal cells; CM, conditioned medium; COL, collagen; HA, sodium hyaluronate; LPS, lipopolysaccharides; PTL, phase-transited lysozyme; qPCR, quantitative PCR; SEM, scanning electron microscopy; TEM, transmission electron microscopy; $\mathrm{Ti}$, titanium; VG, Van Gieson; XPS, X-ray photoelectron spectroscopy. 
A
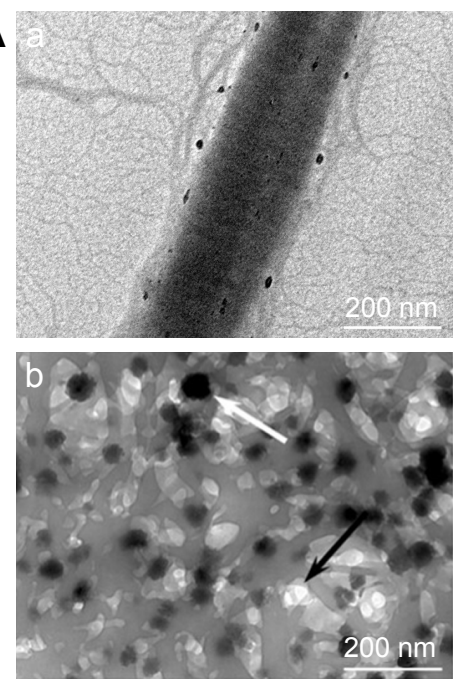

B

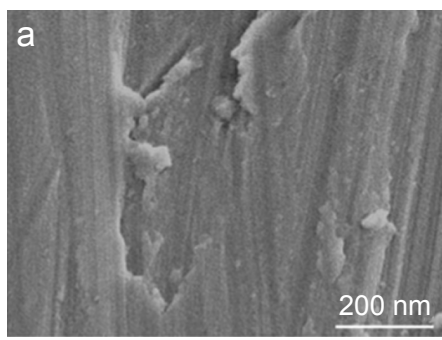

c

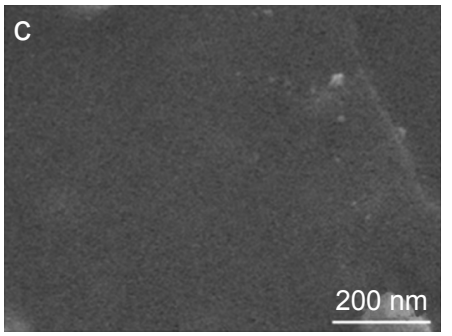

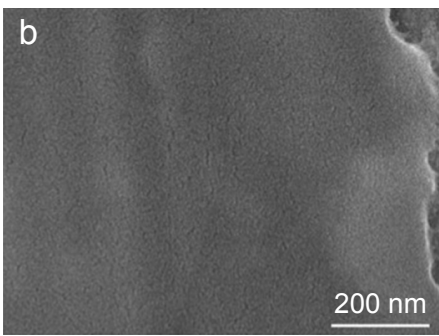

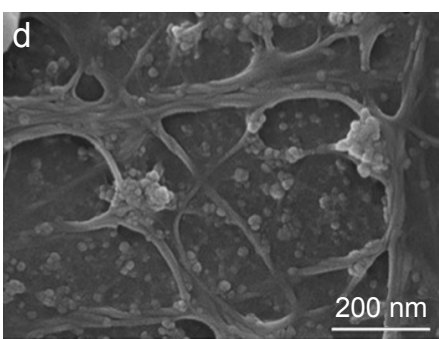

C

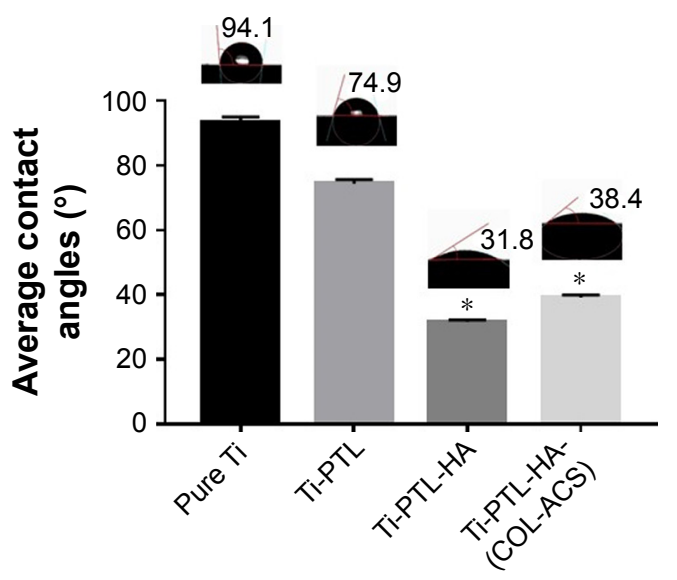

D $\stackrel{0}{\circ}$

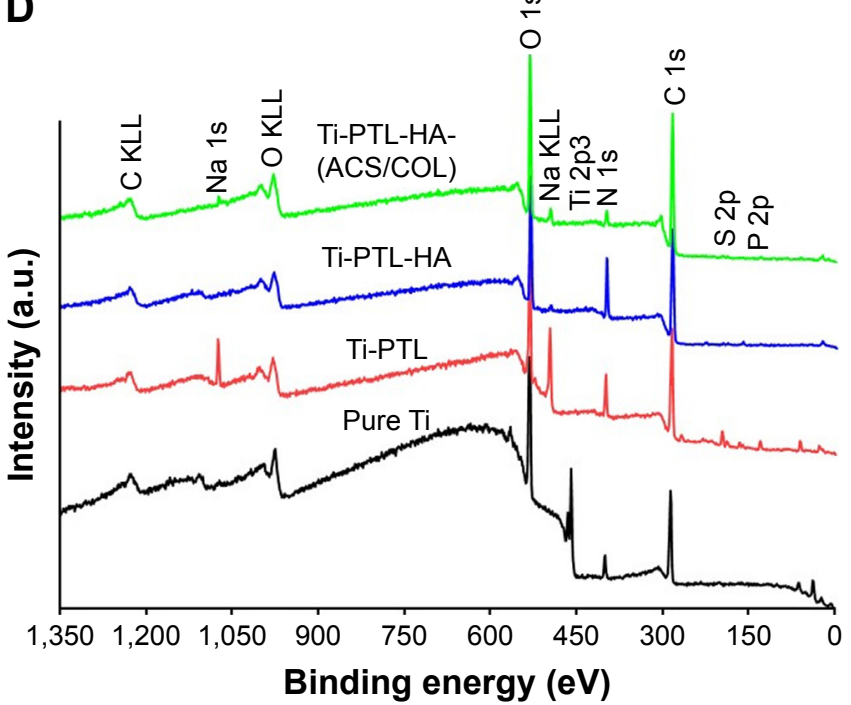

Figure 2 Surface characteristics

Notes: (A) TEM characterization of (a) self-assembled COL I, (b) ACS dispersed in collagen solution; the black arrow indicates the ACS, and the white arrow indicate collagen fibrils. (B) FE-SEM surface morphologies of (a) pure Ti, (b) Ti-PTL, (c) Ti-PTL-HA, (d) Ti-PTL-HA-(COL/ACS). (C) Contact angle of SBF on the surface of the tested samples $(n=3)$. $* P<0.05$, compared with pure Ti. (D) XPS wide-scan spectra of samples after having been immersed in deionized water for 7 days.

Abbreviations: ACS, aspirin-loaded chitosan nanoparticles; COL I, type I collagen; FE-SEM, field-emission scanning electron microscopy; HA, sodium hyaluronate; PTL, phase-transited lysozyme; SBF, simulated body fluid; TEM, transmission electron microscopy; Ti, titanium; XPS, X-ray photoelectron spectroscopy.

of PTL to the Ti substrate. The fall of the S2p, P2p peaks and the rise of the $\mathrm{N} 1 \mathrm{~s}(398.65 \mathrm{eV})$ peak, originating from the HA, indicated that HA was successfully immobilized on the PTL-primed samples. The significant rise of the $\mathrm{C} 1 \mathrm{~s}$
$(284.8 \mathrm{eV})$ peak illustrates that ASA-nanoparticle/assemble collagen was successfully deposited on the sample. Table 2 shows the elemental composition of different samples, as determined by XPS.

Table 2 Elemental composition at the surface of various Ti discs with different treatments as determined by XPS

\begin{tabular}{|l|l|l|l|l|l|l|l|l|}
\hline & $\mathbf{C} \%$ & $\mathbf{N} \%$ & $\mathbf{O} \%$ & $\mathbf{N a} \%$ & $\mathbf{P} \%$ & $\mathbf{S} \%$ & $\mathbf{C l} \%$ & $\mathrm{Ti} \%$ \\
\hline Pure $\mathrm{Ti}$ & $50.4 \mathrm{I} \pm 0.0 \mathrm{I}$ & $2 \mathrm{I} .83 \pm 0.02$ & $35.64 \pm 0.0 \mathrm{I}$ & $0.36 \pm 0.03$ & 0 & $0.19 \pm 0.02$ & $0.18 \pm 0.0 \mathrm{I}$ & $6.74 \pm 0.0 \mathrm{I}$ \\
\hline Ti-PTL & $56.06 \pm 0.02$ & $\mathrm{II} .13 \pm 0.0 \mathrm{I}$ & $2 \mathrm{I} .83 \pm 0.02$ & $5.82 \pm 0.0 \mathrm{I}$ & $2.02 \pm 0.0 \mathrm{I}$ & $\mathrm{I} . \mathrm{II} \pm 0.0 \mathrm{I}$ & $\mathrm{I} .96 \pm 0.02$ & $0.03 \pm 0.02$ \\
\hline Ti-PTL-HA & $59.5 \mathrm{I} \pm 0.0 \mathrm{I}$ & $14.99 \pm 0.03$ & $24.26 \pm 0.0 \mathrm{I}$ & $0.38 \pm 0.02$ & $0.25 \pm 0.02$ & $0.43 \pm 0.03$ & $0.17 \pm 0.03$ & 0 \\
\hline Ti-PTL-HA-(ACS/COL) & $65.75 \pm 0.0 \mathrm{I}$ & $3.89 \pm 0.0 \mathrm{I}$ & $28.66 \pm 0.03$ & $0.94 \pm 0.0 \mathrm{I}$ & $0.36 \pm 0.02$ & $0.2 \mathrm{I} \pm 0.0 \mathrm{I}$ & $0.19 \pm 0.0 \mathrm{I}$ & 0 \\
\hline
\end{tabular}

Abbreviations: ACS, aspirin-loaded chitosan nanoparticles; COL, collagen; HA, sodium hyaluronate; PTL, phase-transited lysozyme; Ti, titanium; XPS, X-ray photoelectron spectroscopy. 
It has been reported that the positive surface potential and moderate hydrophilicity (contact angle $=40^{\circ}-60^{\circ}$ ) of the modified substrates can improve cell spreading, adhesion, and polarity. ${ }^{42,43}$ In this study, the static contact angle measurements using SBF indicated that the modified Ti discs were more hydrophilic than pure Ti discs (Figure 2C). The contact angle on the different stages of the multilayers decreased sharply from $94.9^{\circ} \pm 1.5^{\circ}$ to $38.4^{\circ} \pm 1.3^{\circ}$. Previous studies have demonstrated that hydrophilic surfaces inhibit the adhesion and survival of monocytes, influence the secretion of inflammatory factors to some extent, and improve the osteogenic differentiation of osteoblasts and bone marrow mesenchymal stem cells in vitro and in vivo. ${ }^{44,45}$

In previous studies, NSAIDs affected osteoblast proliferation and osteoclast differentiation and may have adverse effects on bone formation. ${ }^{46-49}$ Therefore, controlling the dose of ASA is the key to application. Compared with other sustained-release drug delivery systems, chitosan nanoparticles have been widely studied for more than a decade. ${ }^{50-52}$ In this study, an emulsion cross-linking method was used to prepare chitosan nanoparticles containing ASA. Although a burst release was observed for ASA on the first day and gradually stabilized after 7 days, which indicated that the ACS performed well in controlling the release of the drug (Figure 3A), the calibration curves of the absorbance of ASA at $280 \mathrm{~nm}$ showed an $\mathrm{R}^{2}=0.997$, and the relatively large amounts of ASA were released into the deionized water. The amount of ASA released at the different intervals (1-7 days) is in the order of $5.0 \mathrm{~mL}$ ASA $>3.0 \mathrm{~mL}$ ASA $>1.5 \mathrm{~mL}$ ASA $>1.0 \mathrm{~mL}$ ASA $>0.5 \mathrm{~mL}$ ASA.

Chitosan is a weakly basic natural polysaccharide with cations that can form polyelectrolyte complexes via the anion from TPP and the amino group of chitosan..$^{53-55}$ The conditions of ACS preparation (concentrations of chitosan and TPP, the ratio of the two, the $\mathrm{pH}$ of the reaction, and the stirring speed) were determined in our previous study. ${ }^{56}$ The TEM images showed that the ACS were spherical in shape with smooth surfaces and were $\sim 100 \mathrm{~nm}$ in size (Figure 2A(b)).

COL I is the most abundant collagen in the body and plays an important role in bone formation. The R-G-D sequence present in the collagen peptide chain can bind to the bone cell surface, inducing cell attachment and promoting the production of specific signals. ${ }^{57}$ Collagen has excellent biocompatibility and low antigenicity and can promote cell growth and differentiation. ${ }^{36,37}$ Therefore, collagen-coated materials can provide a matrix for bone mineralization, promote cell growth, and direct differentiation. ${ }^{58,59}$ The characteristic transverse striations of collagen fibers can be seen under the microscope, indicating that the collagen membrane has completed the self-assembly process (Figure 2A(a)). Moreover, the self-assembled COL I that we prepared also serves as a dispersant for ASA nanoparticles. The field-emission SEM image in Figure 2B shows that the multilayers of HA and COL I loaded with ACS covered the PTL-primed Ti samples.

\section{Behavior of BMSCs on multilayers loaded with ASA}

Cell proliferation is a basic feature of cell life, and its activity can be used to directly assess the cytotoxicity of materials. ${ }^{60}$ The results of the CCK- 8 test showed that, in the process of drug-loaded nanoparticles preparation, the amount of ASA added $(<5.0 \mathrm{~mL})$ can promote cell proliferation (Figure 3B), indicating that the effect of ASA on cell proliferation is
A

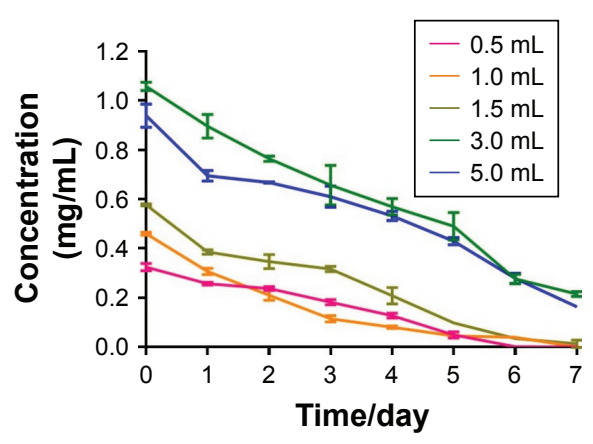

B Growth curve

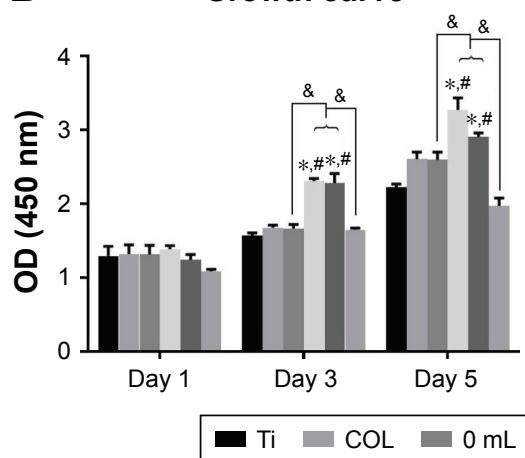

C Relative activity of ALP

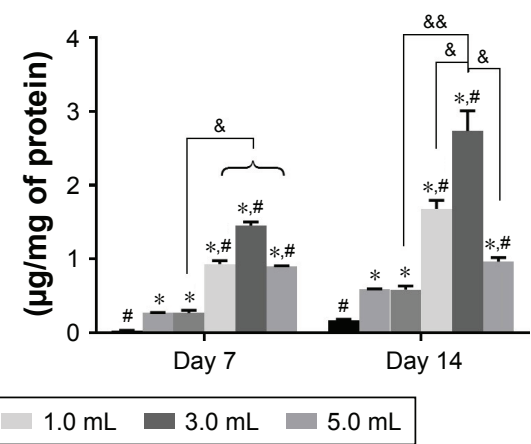

Figure 3 (A) Release curve of different volumes of ASA in the preparation process from multilayers at I, 2, 3, 4, 5, 6, and 7 days (n=3). (B) CCK-8 results of BMSCs after culturing for I, 3 , and 5 days on the different surfaces of Ti discs $(n=3)$. (C) ALP activities of BMSCs cultured for 7 and I4 days on different surfaces of Ti discs ( $n=3$ ). Notes: *denotes the significant difference between Ti and other groups; \#denotes the significant difference between different Ti and other groups; \&denotes the significant difference between different ASA microspheres: $*, \#, \&(P<0.05)$; ${ }^{\& \&}(P<0.0 \mathrm{I})$.

Abbreviations: ASA, aspirin; BMSCs, bone marrow stromal cells; CCK-8, Cell Counting Kit-8; COL, collagen; Ti, titanium. 
concentration-dependent. For the lower concentration range, when the amount of ASA added is $1.0-3.0 \mathrm{~mL}$, the promotion effect on cell proliferation is more obvious.

The ALP kit was used to reflect the osteogenic activity of BMSCs and to evaluate the state of bone metabolism. In the detection of ALP proteins in the cells, the concentration of ASA at the lower concentrations was higher than that in the $5.0 \mathrm{~mL}$ concentrations group (Figure 3C), indicating that the lower ASA concentration group can promote the ALP protein activity of BMSCs. Based on the CCK-8 and ALP activity results above, $3.0 \mathrm{~mL}$ of ACS was used in subsequent experiments.

\section{Synergetic effects of the multifaceted $\mathrm{Ti}$ on anti-inflammatory activity}

In recent years, biological material design paradigms have gradually progressed to include favorable immunomodulatory effects, and materials following this paradigm are expected to regulate the local immune environment and facilitate the interaction between implant and host. ${ }^{61-63} \mathrm{Mac}-$ rophages with plasticity are the bridges linking host immunity and implant in the bone metabolism microenvironment; therefore, they have received the most attention among all the immune cells. ${ }^{64,65}$ At present, the in vitro inflammation model of cells often consists of macrophages using proinflammatory factors such as LPS, TNF- $\alpha$, and IL-1 $\beta \cdot{ }^{66-68}$ LPS acts on tolllike receptors through the LPS-LPS binding protein-sCD14 triple complex to produce inflammatory signals and further activates a series of signal cascades inside/outside the cell, which ultimately causes inflammatory bone loss. ${ }^{69,70}$

Sterile dental implants tend to have mild or moderate inflammatory responses to trauma during implant surgery. Therefore, to mimic the in vitro inflammatory response, in this study, LPS was used to activate the macrophages and was then removed and replaced with a conventional culture medium. The results of this experiment (Figure 4A(a-b)) showed that the level of inflammatory protein markers $\left(\mathrm{NaNO}_{2}\right.$ and TNF- $\alpha$ ) of macrophages in the LPS-induced group was significantly increased compared with that of the group without LPS induction. The result revealed that a macrophage inflammation model was successfully established. Thus, the LPS-mediated inflammation model was used for the detection of materials containing ASA. In this study, under the action of multifaceted coating Ti discs loaded with ASA, the gene expression levels of inflammatory cytokines (IL-6 and TNF- $\alpha$ ) and the level of inflammatory protein marker $\left(\mathrm{NaNO}_{2}\right)$ induced by LPS were lower after 12 hours than those of the blank group without ASA (Figure 4B(a-c)).

The result indicated that the $\mathrm{Ti}$ discs loaded with ASA have immunoregulatory properties for early acute
A a
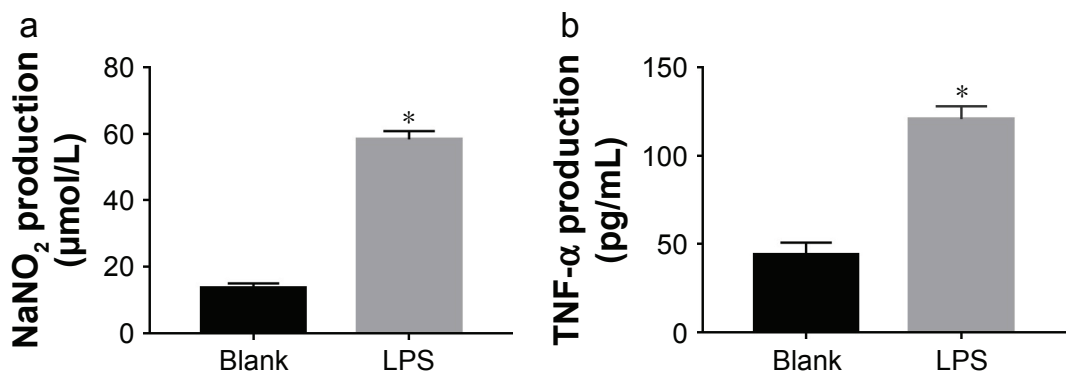

B a

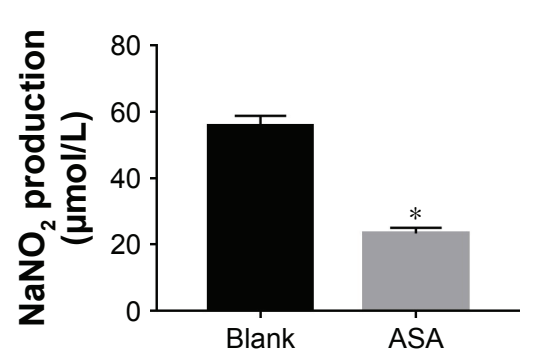

b

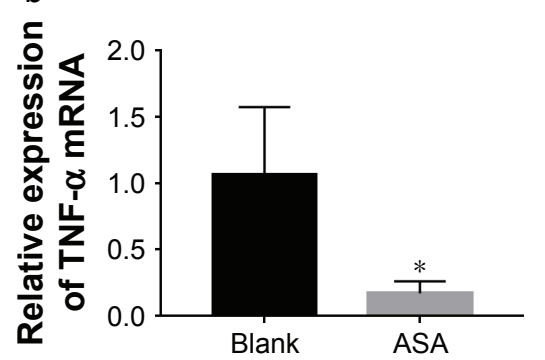

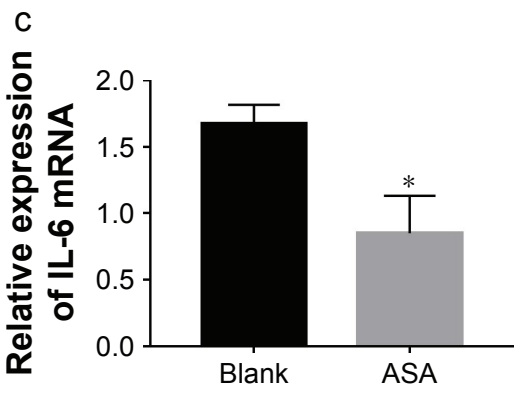

Figure 4 Verifying the success of LPS induction by ELISA and the effect of specimen-conditioned medium of RAW264.7 cells on proinflammatory cytokine expression on Ti surfaces as detected by ELISA and qPCR.

Notes: (A) (a) $\mathrm{NaNO}_{2}$ content, (b) TNF- $\alpha$ content; (B) (a) $\mathrm{NaNO}_{2}$ content, (b) TNF- $\alpha$ gene, and (c) IL-6 gene. (*P<0.05, $\mathrm{n}=3$ ).

Abbreviations: ASA, aspirin; IL-6, interleukin-6; LPS, lipopolysaccharides; $\mathrm{NaNO}_{2}$, nitrite; qPCR, quantitative PCR; TNF- $\alpha$, tumor necrosis factor- $\alpha$. 
inflammatory reactions. In previous studies, NSAIDs were proven to reduce inflammation, which is in agreement with our results. ${ }^{21,71-77}$

\section{BMSCs cultured in different CM of macrophages}

With the proposed concept of "osteoimmunomodulation," researchers have increasingly emphasized the importance of manipulating immune cells to generate a favorable osteoimmune environment for improving the osseointegration of implants. ${ }^{78}$ To investigate the effect of osteoimmunomodulation on osteogenesis in vitro, the CM of macrophages was collected and applied to BMSCs.

\section{Migration and adhesion of BMSCs cultured in CM}

Most of the previous methods for measuring the biocompatibility of biomaterials in vitro did not precisely simulate the environment of the material in vivo and often ignored the effects of host immunity. In the present study, the CM of macrophages was collected, and the environment of the material after being implanted into the body was replicated as much as possible. The ideal artificial bone replacement material not only has good physical and chemical properties, basic biocompatibility, bone conduction performance, and induction performance, but also the basic conditions of an excellent biological material. It can not only provide support for new bone to grow on the surface of a material but also induce the differentiation of pluripotent stem cells into osteoblasts around the implants.

Cell adhesion was assayed by staining with FITC-phalloidin to visualize the F-actin (green) and the nuclei (blue), as shown in Figure 5A. After 12 hours of culture, F-actin extended to a larger polygonal morphology on the surface of Ti containing ASA, while spindle cells were observed on the surface of blank Ti. After 48 hours of culture, pseudopods extended on the surface of blank Ti were significantly shorter than the pseudopods on the surface of prepared $\mathrm{Ti}$, although there was no significant difference in the number of adherent cells in the blank surface.

In the present study, the Transwell coculture model of macrophages was used in the CM of different prepared $\mathrm{Ti}$ samples to study the effect of macrophage secretion products
A
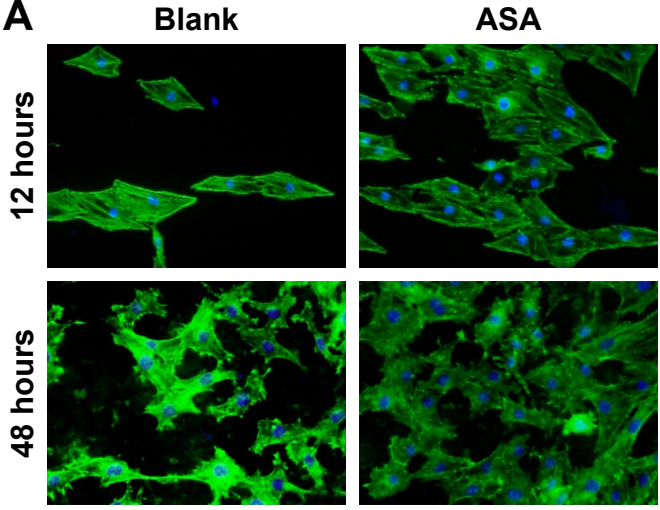

\section{B Cell proliferation and migration}

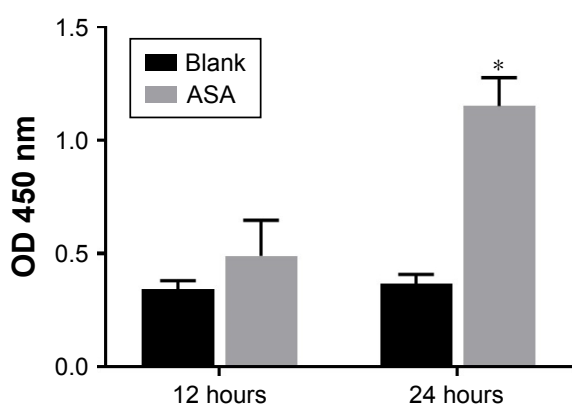

$C_{\text {a }}$

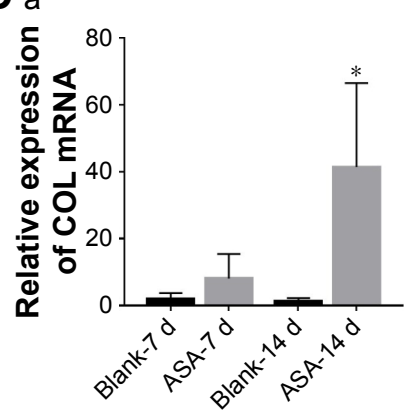

b

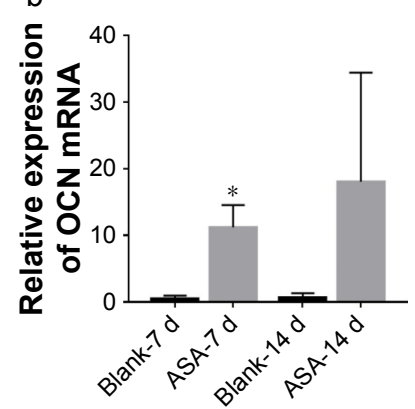

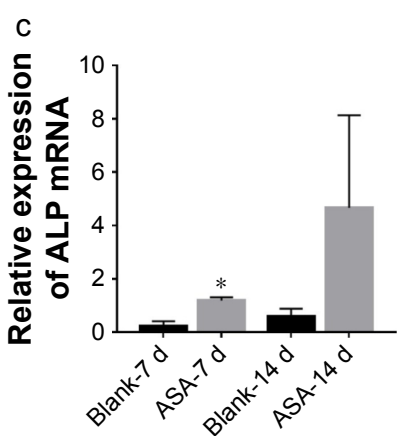

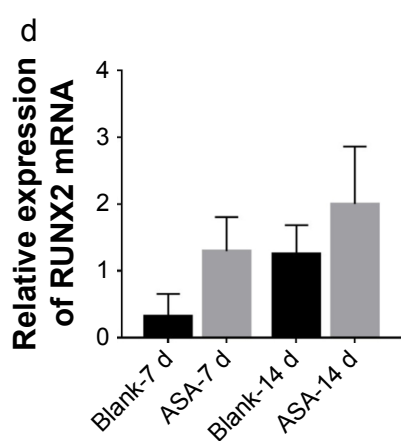

Figure 5 Adhesion, proliferation, migration, and osteogenic differentiation of BMSCs in conditioned medium.

Notes: (A) Adhesion of BMSCs on different Ti surfaces; (B) migration of BMSCs on Ti surfaces in Transwell: greater BMSC migration was observed on the ASA-loaded Ti surface than on the blank surface; (C) effects of the conditioned medium of RAW264.7 cells on osteogenic-related gene expression of BMSCs after culturing for 7 and 14 days, as detected by qPCR: (a) COL I gene, (b) OCN gene, (c) ALP gene, and (d) RUNX2 gene. *P<0.05, $\mathrm{n}=3$.

Abbreviations: ASA, aspirin; BMSCs, bone marrow stromal cells; COL I, type I collagen; OCN, osteocalcin; qPCR, quantitative PCR; RUNX2, runt-related transcription factor $2 ; \mathrm{Ti}$, titanium. 
on the migration of BMSCs. The CCK- 8 result shown in Figure 5B indicates that the number of ASA group was higher than that of the blank group, indicating that multilayering implant-loaded ASA can promote the migration and proliferation of BMSCs.

The homing and osteogenic differentiation of BMSCs determine the speed and efficiency of bone formation and mineralization around the implant and ultimately determine the success of the implant. ${ }^{79}$ The surface property of the implants plays an important role in the adhesion, growth, and differentiation of the cells around it. ${ }^{80}$ The good migration performance of BMSCs in this study can be attributed to the multilayers containing collagen. ${ }^{81}$ In addition, the acute inflammatory reaction induced by the implantation of surgical wounds and the foreign body reaction caused by an implant material can usually regulate the homing and differentiation of BMSCs through the primary immune regulation from ASA. ${ }^{82}$ Therefore, the combination of COL I and ACS coating on implantable Ti discs can improve the migration of BMSC.

\section{Osteogenic differentiation of BMSCs cultured in CM}

Osseointegration is an intricate process of collaboration of multiple cells from different systems. ${ }^{83}$ Interdisciplinary osteoimmunology describes the vital role of immune cells in osteogenesis, and the early immune environment determines the outcome of osseointegration. ${ }^{84}$ To promote early healing of the implant and to improve the effect of osseointegration, the promotion of osteoblast activity and the suppression of osteoclast activity are necessary. The expression of related osteogenic genes (COLI, OCN, ALP, and RUNX2) in BMSCs was upregulated in cells grown in CM collected from macrophages cultured on different $\mathrm{Ti}$ discs with or without ASA (Figure 5C). In the RUNX2 gene expression test, although there is no significant difference in the statistical calculation, the trend can be seen. In brief, these results indicate that the implant containing ASA has surface properties that promote BMSC migration, proliferation, as well as osteogenic differentiation and lead to improved osteogenesis of implants which contributes to osseointegration.

\section{In vivo implantation and histological analysis \\ Histological analysis of inflammation and macrophage distribution}

After a biomaterial is implanted, it is recognized as a foreign body, and the initial adsorption with blood and protein causes acute inflammation. ${ }^{85}$ Macrophages play an important role in the inflammatory response and lead to inflammation and bone healing. ${ }^{86,87}$ Hence, we hypothesized that differences in inflammatory cell infiltration and distribution around the implants are responsible for different osteogenic behaviors observed around the different implanted biomaterials. To test this hypothesis, we observed early inflammatory cell infiltration around the implant using H\&E staining and immunofluorescent staining for $\mathrm{CD}^{+} 8^{+}$cells. As shown in Figure 6, the Blank and ASA groups exhibited a similar inflammatory response at 3 days. There are inflammatory cells, and multinucleated giant cells are scattered in tissues adjacent to the surfaces of the implants.

To clarify the relationship between inflammation and implant material, $\mathrm{CD}^{+} 8^{+}$macrophages were immunofluorescently labelled within the region of the implanted Ti rods. Although the Blank and ASA groups exhibited similar inflammatory responses after 3 days, as shown by the $\mathrm{H} \& \mathrm{E}$ staining, the ASA implants had a smaller macrophage distribution and proportion than the implants in the Blank group

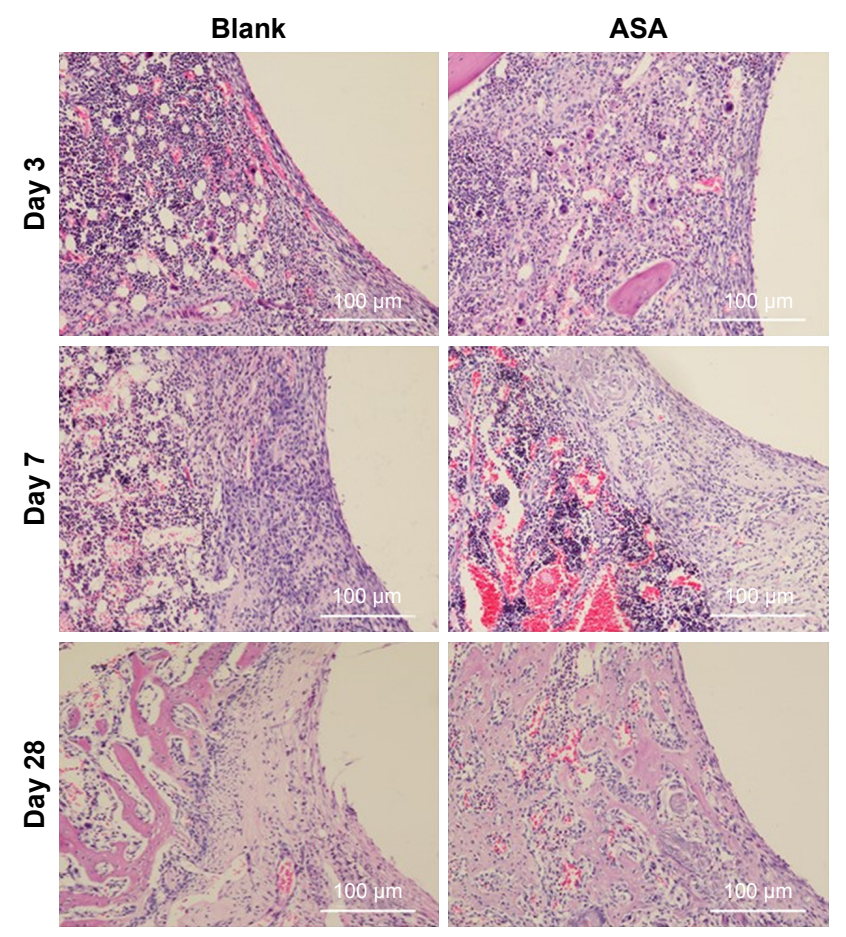

Figure 6 Histological analysis of bone formation in vivo.

Notes: Three days after implantation: the small, deeply stained cells adjacent to the surface of the implants were inflammatory cells infiltrating the tissues. In addition, multinucleated giant cells were scattered in the tissues. There was little difference between the two groups. Seven days after implantation: the inflammatory cells infiltrating the implant surface were significantly more numerous in the blank group than in the ASA group, and bone matrix began to appear in the ASA group. Twenty-eight days after implantation: short, cubic preosteoblasts arranged around the trabecular bone were well formed around the ASA-loaded implants; at the same time, newly formed, small blood vessels were scattered in the trabecular bone at the ASA-loaded surface. Abbreviation: ASA, aspirin. 
(Figure 7A). In addition, the implants in the ASA group significantly inhibited inflammatory cell infiltration after 7 days (Figure 7B). This difference is more pronounced 7 days after implantation, indicating that the implants loaded with ASA have an anti-inflammatory effect.

\section{Histological analysis of bone formation}

To observe in vivo osseointegration of the prepared $\mathrm{Ti}$ implants, the bone implant interface was observed on

A

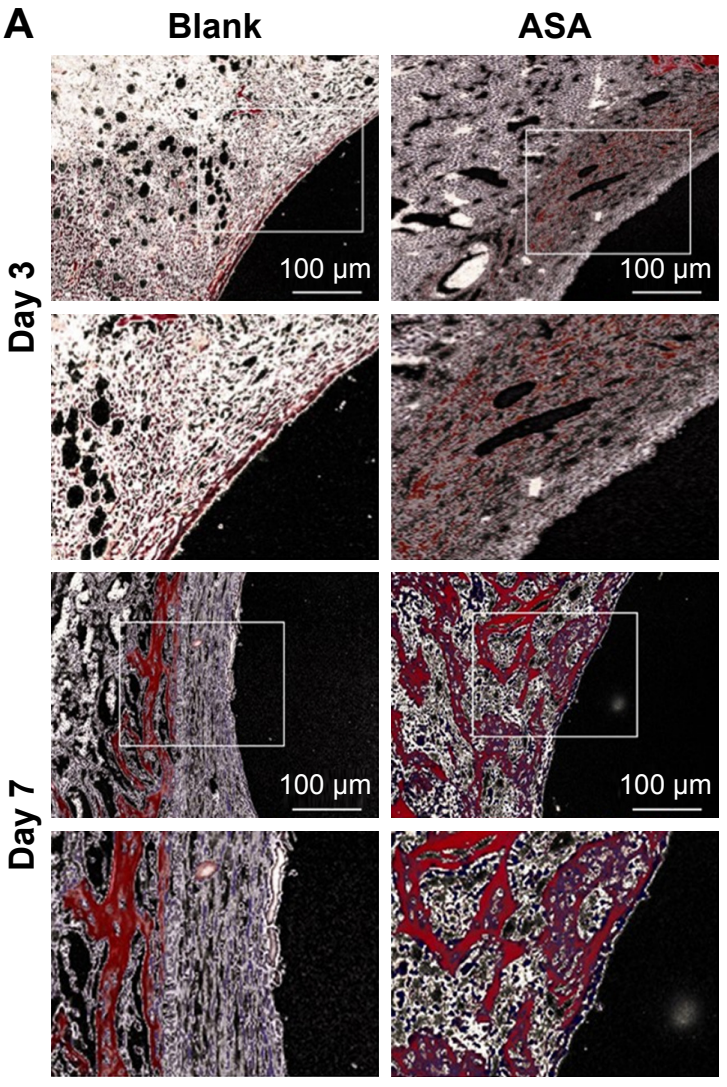

B

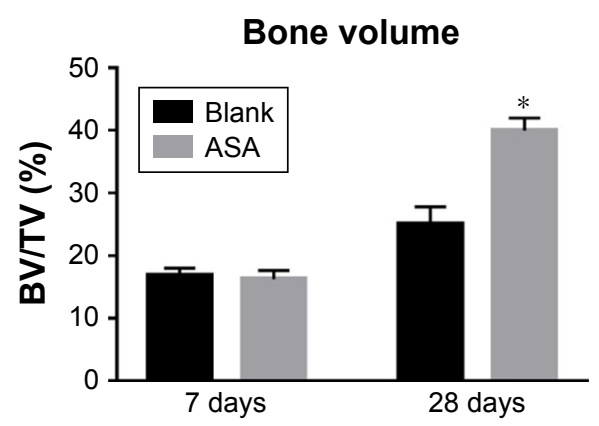

Figure 7 VG staining: representative undecalcified histological images of new bone formation around and within the implants detected by VG staining.

Notes: (A) The bone tissue is stained red, and the fibrous tissue is stained yellow and white. (B) Histomorphometric measurement of bone volume fraction within the implants showed that the ASA group has the highest amount of new bone ingrowth, which is higher than that in the blank group $\left({ }^{*} P<0.05, n=3\right)$.

Abbreviations: ASA, aspirin; BV/TV, the new bone volume fraction; VG staining, Van Gieson staining.
H\&E-stained sections and VG-stained sections of explanted samples (Figure 8). At 28 days after implantation (Figure 6), short and cubic preosteoblasts arranged around the trabecular bone were well formed around the ASA-loaded implants; at the same time, newly formed small blood vessels are scattered in the trabecular bone. Osteogenesis and angiogenesis are intricately coordinated during bone formation. ${ }^{88}$ The ASA group has a higher new bone volume fraction than that on the implants of the blank group, which has no mature bone, and new bone matrix was observed around the implants, as shown in Figure 7B. The implants loaded with ASA demonstrated a greater bone-induction effect than those in the Blank group, with the same distance from the implant surface as a baseline at all time points.

Implants enter the body as an exogenous substance and cause acute inflammatory reaction, and they are surrounded by infiltrating inflammatory cells. However, $\sim 7$ days after implantation, inflammation has different outcomes in different material environments. The inflammatory response
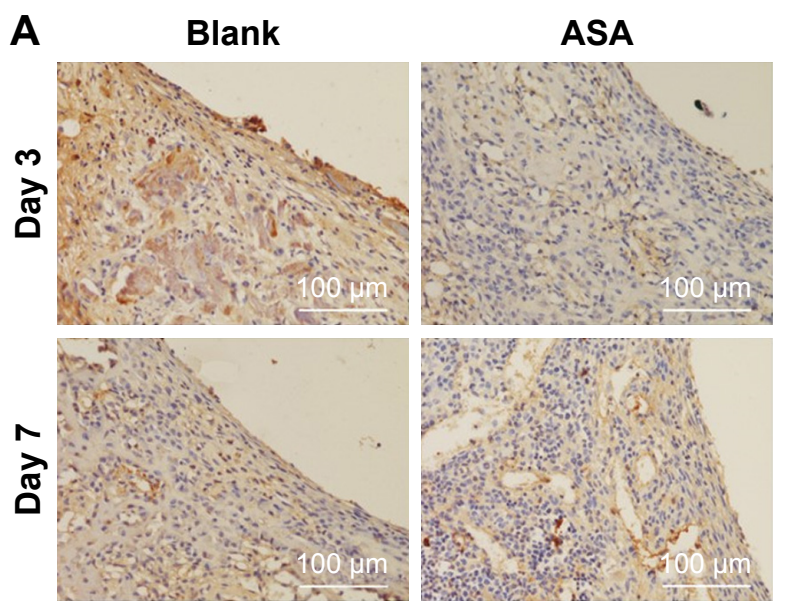

\section{B}

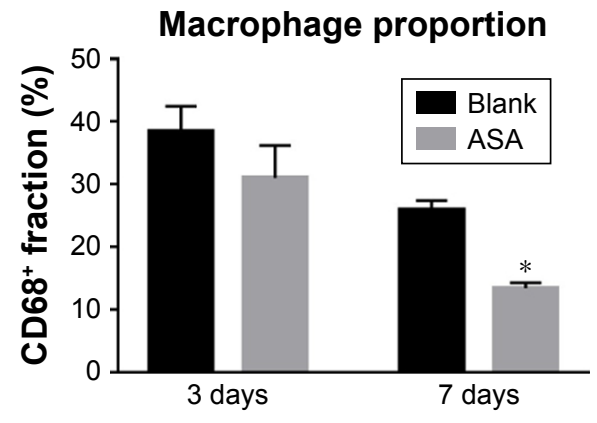

Figure 8 Immunofluorescence staining for $\mathrm{CD} 68^{+}$cells.

Notes: A brownish yellow color indicates positive macrophage infiltration. (A) The numbers of inflammatory cells around the surfaces of two groups did not differ significantly on the 3rd day. On the 7th day, fewer inflammatory cells were observed on the surfaces of the ASA group than on the surfaces of the blank group. (B) The ASA group elicited a lower proportion (\%) of $C D 68^{+}$macrophages than the blank group. $* \mathrm{P}<0.05, \mathrm{n}=3$.

Abbreviation: ASA, aspirin. 
to the implants loaded with ASA is lower than that to those in the Blank group, which also corresponds to the difference in the volume of bone formed around the implants. Overall, our results provide evidence that there is a key biological difference between the in vivo osteoimmunomodulatory response of biomaterials and in vitro experimental conditions.

When biomaterials are implanted in vivo as a foreign body, the process of osseointegration often comprises three stages. In the first stage, tissue adjacent to the biomaterial is affected by the physicochemical properties of the surface of the implant, triggering a host immune response. ${ }^{61}$ The surfaces of the implants loaded with ASA can downregulate the expression of inflammatory cytokines, eliciting favorable osteoimmunomodulation, which lays the foundation for subsequent reactions and guides tissue healing. In the second stage, blood vessels begin to form on the surface of the implant, and BMSCs are also recruited to the surface and accrue around the newly formed vasculature; subsequently, differentiated osteoblasts participate in the dynamic balance of the bone microenvironment. ${ }^{3}$ In this study, the implants loaded with ASA were shown to enhance BMSC adhesion and proliferation and promote osteogenic differentiation both in vitro and in vivo. Over time, collagen fibers secreted by the cells accumulate around the implants, and the loosely organized woven bone gradually remodels to an organized lamellar structure, ultimately completing the process of osseointegration. ${ }^{89}$

\section{Conclusion}

The present study demonstrated the feasibility of loading ACS into HA/COL PEMs on Ti surfaces primed with PTL. The PTL technology has advantages of simple design, low cost, high safety, and easy accessibility; thus, it can be used as an effective method to activate the surfaces of implants and lay the foundation for the subsequent introduction of ASA. The in vitro and in vivo results suggest that the ASA-loaded Ti surface has good performance in osteoimmunomodulation and can further improve osteogenesis, thus potentially providing a superior strategy for the surface modulation of biomedical implants.

\section{Acknowledgments}

This study was supported by a grant from Scientific Research Fund of Tianjin Medical University (No 2016KYZQ07). We thank Zinan Chen for his contribution.

\section{Disclosure}

The authors report no conflicts of interest in this work.

\section{References}

1. Sul YT. The significance of the surface properties of oxidized titanium to the bone response: special emphasis on potential biochemical bonding of oxidized titanium implant. Biomaterials. 2003;24(22): 3893-3907.

2. Bose S, Fielding G, Tarafder S, Bandyopadhyay A. Understanding of dopant-induced osteogenesis and angiogenesis in calcium phosphate ceramics. Trends Biotechnol. 2013;31(10):594-605.

3. Bai L, Du Z, Du J, et al. A multifaceted coating on titanium dictates osteoimmunomodulation and osteo/angio-genesis towards ameliorative osseointegration. Biomaterials. 2018;162:154-169.

4. Jia Z, Xiu P, Li M, et al. Bioinspired anchoring AgNPs onto micronanoporous $\mathrm{TiO} 2$ orthopedic coatings: Trap-killing of bacteria, surface-regulated osteoblast functions and host responses. Biomaterials. 2016;75:203-222.

5. Liu Y, Wang L, Kikuiri T, et al. Mesenchymal stem cell-based tissue regeneration is governed by recipient $\mathrm{T}$ lymphocytes via IFN- $\gamma$ and TNF- $\alpha$. Nat Med. 2011;17(12):1594-1601.

6. Kakugawa Y, Wada T, Yamaguchi K, et al. Up-regulation of plasma membrane-associated ganglioside sialidase (NEU3) in human colon cancer and its involvement in apoptosis suppression. Proceedings of the National Academy of Sciences. 2002;99(16):10718-10723.

7. Takayanagi H. Osteoimmunology: shared mechanisms and crosstalk between the immune and bone systems. Nat Rev Immunol. 2007; 7(4):292-304.

8. Walsh MC, Kim N, Kadono Y, et al. Osteoimmunology: interplay between the immune system and bone metabolism. Annu Rev Immunol. 2006;24(1):33-63.

9. Takayanagi H. Osteoimmunology: shared mechanisms and crosstalk between the immune and bone systems. Nat Rev Immunol. 2007;7(4): 292-304.

10. Brown BN, Ratner BD, Goodman SB, Amar S, Badylak SF. Macrophage polarization: an opportunity for improved outcomes in biomaterials and regenerative medicine. Biomaterials. 2012;33(15):3792-3802.

11. Omar OM, Granéli C, Ekström K, et al. The stimulation of an osteogenic response by classical monocyte activation. Biomaterials. 2011; 32(32):8190-8204.

12. Chen S, Jones JA, Xu Y, Low HY, Anderson JM, Leong KW. Characterization of topographical effects on macrophage behavior in a foreign body response model. Biomaterials. 2010;31(13):3479-3491.

13. Chen Z, Yuen J, Crawford R, Chang J, Wu C, Xiao Y. The effect of osteoimmunomodulation on the osteogenic effects of cobalt incorporated $\beta$-tricalcium phosphate. Biomaterials. 2015;61:126-138.

14. Li Y, Luo Z, Xu X, et al. Aspirin enhances the osteogenic and antiinflammatory effects of human mesenchymal stem cells on osteogenic BFP-1 peptide-decorated substrates. J Mater Chem B. 2017;5(34): 7153-7163.

15. Preshaw PM, Hefti AF, Jepsen S, Etienne D, Walker C, Bradshaw MH. Subantimicrobial dose doxycycline as adjunctive treatment for periodontitis. A review. J Clin Periodontol. 2004;31(9):697-707.

16. Simonet WS, Lacey DL, Dunstan CR, et al. Osteoprotegerin: a novel secreted protein involved in the regulation of bone density. Cell. 1997;89(2):309-319.

17. Vardar S, Buduneli E, Baylas H, Berdeli AH, Buduneli N, Atilla G. Individual and combined effects of selective cyclooxygenase-2 inhibitor and omega-3 fatty acid on endotoxin-induced periodontitis in rats. J Periodontol. 2005;76(1):99-106.

18. Barragan-Adjemian C, Lausten L, Ang DB, Johnson M, Katz J, Bonewald LF. Bisphosphonate-related osteonecrosis of the jaw: model and diagnosis with cone beam computerized tomography. Cells Tissues Organs. 2009;189(1-4):284-288.

19. Yusuf S, Zhao F, Mehta SR, et al. Effects of clopidogrel in addition to aspirin in patients with acute coronary syndromes without ST-segment elevation. N Engl J Med. 2001;345(7):494-502.

20. Fuster V, Sweeny JM. Aspirin: a historical and contemporary therapeutic overview. Circulation. 2011;123(7):768-778. 
21. Du J, Mei S, Guo L, et al. Platelet-rich fibrin/aspirin complex promotes alveolar bone regeneration in periodontal defect in rats. $J$ Periodontal Res. 2018;53(1):47-56.

22. Tang J, Xiong J, Wu T, et al. Aspirin treatment improved mesenchymal stem cell immunomodulatory properties via the 15d-PGJ2/PPAR $\gamma /$ TGF- $\beta 1$ pathway. Stem Cells Dev. 2014;23(17):2093-2103.

23. Cao Y, Xiong J, Mei S, et al. Aspirin promotes bone marrow mesenchymal stem cell-based calvarial bone regeneration in mini swine. Stem Cell Res Ther. 2015;6(1):210.

24. Du M, Pan W, Duan X, Yang P, Ge S. Lower dosage of aspirin promotes cell growth and osteogenic differentiation in murine bone marrow stromal cells. J Dent Sci. 2016;11(3):315-322.

25. Zeng YP, Yang C, Li Y, et al. Aspirin inhibits osteoclastogenesis by suppressing the activation of NF-KB and MAPKs in RANKL-induced RAW264.7 cells. Mol Med Rep. 2016;14(3):1957-1962.

26. Liu Y, Fang S, Li X, et al. Aspirin inhibits LPS-induced macrophage activation via the NF-KB pathway. Sci Rep. 2017;7(1):11549.

27. Mercanzini A, Reddy ST, Velluto D, et al. Controlled release nanoparticle-embedded coatings reduce the tissue reaction to neuroprostheses. J Control Release. 2010;145(3):196-202.

28. Shi Y, Wan A, Shi Y, Zhang Y, Chen Y. Experimental and mathematical studies on the drug release properties of aspirin loaded chitosan nanoparticles. BioMed. 2014;2014(6):1-8.

29. Wang D, Ha Y, Gu J, Li Q, Zhang L, Yang P. 2D protein supramolecular Nanofilm with exceptionally large area and emergent functions. $A d v$ Mater. 2016;28(34):7414-7423.

30. Wang SS, Liu KN, Lu YC. Amyloid fibrillation of hen egg-white lysozyme is inhibited by TCEP. Biochem Biophys Res Commun. 2009;381(4):639-642.

31. Liu R, Zhao J, Han Q, et al. One-step assembly of a biomimetic biopolymer coating for particle surface engineering. Adv Mater. 2018; 30(38):e1802851.

32. Wu Z, Yang P. Simple multipurpose surface Functionalization by phase Transited protein adhesion. Advanced Materials Interfaces. 2015;2(2):1400401.

33. Ha Y, Yang J, Tao F, et al. Phase-Transited lysozyme as a universal route to bioactive hydroxyapatite crystalline film. Advanced Functional Materials. 2018;28(4):1704476.

34. Zhong X, Song Y, Yang P, et al. Titanium surface priming with Phase-Transited lysozyme to establish a silver Nanoparticle-Loaded Chitosan/Hyaluronic acid antibacterial multilayer via layer-by-layer self-assembly. PLoS One. 2016;11(1):e0146957.

35. Guan B, Wang $\mathrm{H}, \mathrm{Xu} \mathrm{R}$, et al. Establishing antibacterial multilayer films on the surface of direct metal laser Sintered titanium primed with Phase-Transited lysozyme. Sci Rep. 2016;6(1):36408.

36. Shanmugam G, Reddy SMM, Madhan B, Rao JR. Method of addition of acetonitrile influences the structure and stability of collagen. Process Biochemistry. 2014;49(2):210-216.

37. Li GY, Fukunaga S, Takenouchi K, Nakamura F. Comparative study of the physiological properties of collagen, gelatin and collagen hydrolysate as cosmetic materials. Int J Cosmet Sci. 2005;27(2):101-106.

38. Frantz C, Stewart KM, Weaver VM. The extracellular matrix at a glance. J Cell Sci. 2010;123(Pt 24):4195-4200.

39. Wang Y, van Manh N, Wang H, Zhong X, Zhang X, Li C. Synergistic intrafibrillar/extrafibrillar mineralization of collagen scaffolds based on a biomimetic strategy to promote the regeneration of bone defects. Int J Nanomedicine. 2016;11:2053-2067.

40. Wu Z, Yang P. Simple multipurpose surface functionalization by phase transited protein adhesion. Adv Mater Interfaces. 2015;2(2):1400401.

41. Li C, Qin R, Liu R, Miao S, Yang P. Functional amyloid materials at surfaces/interfaces. Biomater Sci. 2018;6(3):462-472.

42. Pandey LM, Pattanayek SK. Hybrid surface from self-assembled layer and its effect on protein adsorption. Applied Surface Science. 2011; 257(10):4731-4737.

43. Chang H-Y, Huang C-C, Lin K-Y, et al. Effect of surface potential on NIH3T3 cell adhesion and proliferation. $J$ Phys Chem $C$. 2014;118(26):14464-14470.
44. Brodbeck WG, Patel J, Voskerician G, et al. Biomaterial adherent macrophage apoptosis is increased by hydrophilic and anionic substrates in vivo. Proc Natl Acad Sci. 2002;99(16):10287-10292.

45. Ivanovski S, Hamlet S, Salvi GE, et al. Transcriptional profiling of osseointegration in humans. Clin Oral Implants Res. 2011;22(4): 373-381.

46. Pountos I, Georgouli T, Calori GM, Giannoudis PV. Do nonsteroidal anti-inflammatory drugs affect bone healing? A critical analysis. Sci World J. 2012;2012(2):1-14.

47. Müller M, Raabe O, Addicks K, Wenisch S, Arnhold S. Effects of nonsteroidal anti-inflammatory drugs on proliferation, differentiation and migration in equine mesenchymal stem cells. Cell Biol Int. 2011;35(3): 235-248.

48. Elkhouli AM. The efficacy of host response modulation therapy (omega-3 plus low-dose aspirin) as an adjunctive treatment of chronic periodontitis (clinical and biochemical study). $J$ Periodontal Res. 2011;46(2):261-268

49. Shiloah J, Bland PS, Scarbecz M, Patters MR, Stein SH, Tipton DA. The effect of long-term aspirin intake on the outcome of non-surgical periodontal therapy in smokers: a double-blind, randomized pilot study. J Periodontal Res. 2014;49(1):102-109.

50. Agnihotri SA, Aminabhavi TM. Controlled release of clozapine through chitosan microparticles prepared by a novel method. J Control Release. 2004;96(2):245-259.

51. Sokker HH, Abdel Ghaffar AM, Gad YH, Aly AS, Ghaffar AMA, Asi A. Synthesis and characterization of hydrogels based on grafted chitosan for the controlled drug release. Carbohydrate Polymers. 2009;75(2):222-229.

52. Agnihotri SA, Mallikarjuna NN, Aminabhavi TM. Recent advances on chitosan-based micro- and nanoparticles in drug delivery. $J$ Control Release. 2004;100(1):5-28.

53. Wang AH, Chen XG, Liu CS, Meng XH, Yu LJ, Wang H. Preparation and characteristics of chitosan microspheres in different acetylation as drug carrier system. J Microencapsul. 2009;26(7): 593-602.

54. Dass CR, Choong PF. Chitosan-mediated orally delivered nucleic acids: a gutful of gene therapy. J Drug Target. 2008;16(4):257-261.

55. Zheng AP, Wang JC, Lu WL, et al. Thymopentin-loaded $\mathrm{pH}$-sensitive chitosan nanoparticles for oral administration: preparation, characterization, and pharmacodynamics. J Nanosci Nanotechnol. 2006; 6(9-10):2936-2944.

56. Zhang J, Ma S, Liu Z, et al. Guided bone regeneration with asymmetric collagen-chitosan membranes containing aspirin-loaded chitosan nanoparticles. Int J Nanomedicine. 2017;12:8855-8866.

57. Felgueiras HP, Sommerfeld SD, Murthy NS, Kohn J, Migonney V, Poly MV. Poly(NaSS) functionalization modulates the conformation of fibronectin and collagen type I to enhance osteoblastic cell attachment onto Ti6Al4V. Langmuir. 2014;30(31):9477-9483.

58. Liang $\mathrm{C}$, Zhong $\mathrm{X}$, Wang $\mathrm{H}$, et al. Femtosecond laser induced micropatterns and in-situ deposition of $\mathrm{Ca} / \mathrm{P}$ phase and collagen on Ti surface. Mater Chem Phys. 2015;158:115-120.

59. Schulz MC, Korn P, Stadlinger B, et al. Coating with artificial matrices from collagen and sulfated hyaluronan influences the osseointegration of dental implants. J Mater Sci Mater Med. 2014;25(1):247-258.

60. Ravichandran R, Sundarrajan S, Venugopal JR, Mukherjee S, Ramakrishna S. Applications of conducting polymers and their issues in biomedical engineering. JR Soc Interface. 2010;7(suppl_5):S559-579.

61. Franz S, Rammelt S, Scharnweber D, Simon JC. Immune responses to implants - a review of the implications for the design of immunomodulatory biomaterials. Biomaterials. 2011;32(28):6692-6709.

62. Williams DF. On the nature of biomaterials. Biomaterials. 2009; 30(30):5897-5909.

63. Veiseh O, Doloff JC, Ma M, et al. Size- and shape-dependent foreign body immune response to materials implanted in rodents and non-human primates. Nat Mater. 2015;14(6):643-651.

64. Xia Z, Triffitt JT. A review on macrophage responses to biomaterials. Biomed Mater. 2006;1(1):R1-R9. 
65. Sridharan R, Cameron AR, Kelly DJ, Kearney CJ, O’Brien FJ. Biomaterial based modulation of macrophage polarization: a review and suggested design principles. Materials Today. 2015;18(6):313-325.

66. Mills CD, Kincaid K, Alt JM, Heilman MJ, Hill AM. M-1/M-2 macrophages and the Th1/Th2 paradigm. J Immunol. 2000;164(12):6166-6173.

67. Mantovani A, Biswas SK, Galdiero MR, Sica A, Locati M. Macrophage plasticity and polarization in tissue repair and remodelling. J Pathol. 2013;229(2):176-185.

68. Meng F, Lowell CA. Lipopolysaccharide (LPS)-induced macrophage activation and signal transduction in the absence of Src-family kinases Hck, Fgr, and Lyn. J Exp Med. 1997;185(9):1661-1670.

69. Chung YH, Chang EJ, Kim SJ, et al. Lipopolysaccharide from Prevotella nigrescens stimulates osteoclastogenesis in cocultures of bone marrow mononuclear cells and primary osteoblasts. J Periodontal Res. 2006;41(4):288-296.

70. Xing Q, de Vos P, Faas MM, Ye Q, Ren Y. LPS promotes preosteoclast activity by up-regulating CXCR4 via TLR-4. J Dent Res. 2011;90(2):157-162.

71. Orihuela R, Mcpherson CA, Harry GJ. Microglial M1/M2 polarization and metabolic states. Br J Pharmacol. 2016;173(4):649-665.

72. Llorens-Martín M, Jurado-Arjona J, Bolós M, Pallas-Bazarra N, Ávila J. Forced swimming sabotages the morphological and synaptic maturation of newborn granule neurons and triggers a unique pro-inflammatory milieu in the hippocampus. Brain Behav Immun. 2016;53:242-254.

73. Yamaza T, Miura Y, Bi Y, et al. Pharmacologic stem cell based intervention as a new approach to osteoporosis treatment in rodents. PLoS One. 2008;3(7):e2615.

74. Kwon MS, Shim EJ, Seo YJ, et al. Effect of aspirin and acetaminophen on proinflammatory cytokine-induced pain behavior in mice. Pharmacology. 2005;74(3):152-156.

75. Buckland M, Jago CB, Fazekasova H, et al. Aspirin-treated human DCs up-regulate ILT-3 and induce hyporesponsiveness and regulatory activity in Responder T cells. Am J Transplant. 2006;6(9): 2046-2059.

76. Dekel S, Lenthall G, Francis MJ. Release of prostaglandins from bone and muscle after tibial fracture. An experimental study in rabbits. J Bone Joint Surg Br. 1981;63-B(2):185-189.
77. Liu H, Li W, Liu Y, Zhang X, Zhou Y. Co-administration of aspirin and allogeneic adipose-derived stromal cells attenuates bone loss in ovariectomized rats through the anti-inflammatory and chemotactic abilities of aspirin. Stem Cell Res Ther. 2015;6(1):200.

78. Chen Z, Klein T, Murray RZ, et al. Osteoimmunomodulation for the development of advanced bone biomaterials. Materials Today. 2016;19(6):304-321.

79. Bianco P, Robey PG, Simmons PJ. Mesenchymal stem cells: revisiting history, concepts, and assays. Cell Stem Cell. 2008;2(4):313-319.

80. Webster TJ, Ergun C, Doremus RH, Siegel RW, Bizios R. Enhanced osteoclast-like cell functions on nanophase ceramics. Biomaterials. 2001;22(11):1327-1333.

81. Nakajima I, Muroya S, Tanabe R, Chikuni K. Extracellular matrix development during differentiation into adipocytes with a unique increase in type V and VI collagen. Biol Cell. 2002;94(3):197-203.

82. Gerstenfeld LC, Edgar CM, Kakar S, Jacobsen KA, Einhorn TA. Osteogenic Growth Factors and Cytokines and Their Role in Bone Repair. 1970.

83. Agarwal R, García AJ. Biomaterial strategies for engineering implants for enhanced osseointegration and bone repair. Adv Drug Deliv Rev. 2015;94:53-62.

84. Miron RJ, Bosshardt DD. OsteoMacs: key players around bone biomaterials. Biomaterials. 2016;82:1-19.

85. Brown BN, Badylak SF. Expanded applications, shifting paradigms and an improved understanding of host-biomaterial interactions. Acta Biomater. 2013;9(2):4948-4955.

86. Mantovani A, Sica A, Sozzani S, Allavena P, Vecchi A, Locati M. The chemokine system in diverse forms of macrophage activation and polarization. Trends Immunol. 2004;25(12):677-686.

87. Gordon S, Martinez FO. Alternative activation of macrophages: mechanism and functions. Immunity. 2010;32(5):593-604.

88. Chang PC, Chong LY, Dovban AS, et al. Sequential platelet-derived growth factor-simvastatin release promotes dentoalveolar regeneration. Tissue Eng Part A. 2014;20(1-2):356-364.

89. Berglundh T, Abrahamsson I, Lang NP, Lindhe J. De novo alveolar bone formation adjacent to endosseous implants. A model study in the dog. Clin Oral Implan Res. 2003;14(3):251-262.
International Journal of Nanomedicine

\section{Publish your work in this journal}

The International Journal of Nanomedicine is an international, peerreviewed journal focusing on the application of nanotechnology in diagnostics, therapeutics, and drug delivery systems throughout the biomedical field. This journal is indexed on PubMed Central,

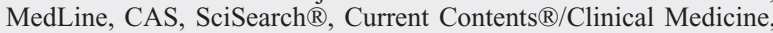

\section{Dovepress}

Journal Citation Reports/Science Edition, EMBase, Scopus and the Elsevier Bibliographic databases. The manuscript management system is completely online and includes a very quick and fair peer-review system, which is all easy to use. Visit http://www.dovepress.com/ testimonials.php to read real quotes from published authors. 